\title{
Antigenic Relationships Among Species of Mycobacterium Studied by Fused Rocket Immunoelectrophoresis
}

\author{
SOTIROS D. CHAPARAS, TERESA BROWN, AND IRIS HYMAN \\ Bureau of Biologics, Food and Drug Administration, Bethesda, Maryland 20014
}

\begin{abstract}
The fused rocket immunoelectrophoresis procedure is a sensitive procedure for detecting precipitating antigens in extracts of mycobacteria. A number of preparations can be run simultaneously and in parallel with the production of lines of identity. The increased sensitivity and facility of identification make this procedure a very promising one in the study of taxonomic relationships.
\end{abstract}

Analysis of antigenic sharing among mycobacteria is a valuable taxonomic tool. However, our knowledge of antigen relationships among microorganisms is limited by several factors, including the quality of the antigenic preparation and the antibody used for making comparisons, and the sensitivity of the procedure used to detect and relate antigens. All studies, including the one presented here, suffer to various degrees in each of these limitations.

In the studies to follow the large numbers of antigens in mycobacterial extracts have been compared by use of fused rocket immunoelectrophoresis (FRIEP). Insight has been gained into the complex degree of antigenic sharing which has hampered efforts to fractionate single monospecific antigens from these species. In addition, the FRIEP procedure presents itself as a powerful tool which can be of value in suggesting relationships among species and in harmonizing existing taxonomic difficulties.

\section{MATERIALS AND METHODS}

Mycobacterial sonicates. The mycobacteria used in this study were obtained from the Trudeau Mycobacterial Collection (TMC) (Trudeau Institute, Saranac Lake, N.Y.) and include the species shown in Table 1.

Organisms were grown on asparagine-glycerol synthetic medium (5). Growth of all organisms was at $35^{\circ} \mathrm{C}$ for periods ranging from 1 to 5 weeks. The organisms were harvested from stationary cultures in 1-liter Blake bottles containing $200 \mathrm{ml}$ of medium a few days after a complete pellicle had formed on the surface. Organisms were washed three times with distilled water by centrifugation, and the pellet was suspended in an equal volume of distilled water. The organisms were then disrupted by sonic treatment with a Branson Sonifier (model W-350) at maximum output for $15 \mathrm{~min}$. Disruption of organisms was confirmed microscopically and by a decrease in the mass of pellet. The sonic extract was centrifuged at about $60,000 \times g$ for $30 \mathrm{~min}$. The supernatant was filtered through a $0.45-\mu \mathrm{m}$-pore-size Nalgene filter, distributed in aliquots, and frozen. Attempts had been made to dialyze the extracts and freeze-dry them, but this procedure resulted in extracts that produced fewer precipitin bands.

Antisera. Rabbits were initially immunized by the injection of a total of $1.0 \mathrm{ml}$ of a Freund emulsion containing homologous organism and sonic extract. The emulsion was prepared by repeated passage through a double-hub needle connected to $6.0-\mathrm{ml}$ syringes. Each milliliter consisted of $0.5 \mathrm{ml}$ of Arlacel A:Drakeol 6VR (35:65), $0.2 \mathrm{ml}$ of sonic extract, $0.3 \mathrm{ml}$ of isotonic saline, and $10 \mathrm{mg}$ of homologous organism. The homologous organism had been harvested during the log phase of growth, killed by steaming for $10 \mathrm{~min}$ at $100^{\circ} \mathrm{C}$, and dried in $35^{\circ} \mathrm{C}$ incubator. The organisms were ground with a mortar and pestle before incorporation into the emulsion. Each rabbit received $0.1 \mathrm{ml}$ in each of the four footpads and into six symmetrically distributed lateral sites. Every 2 weeks thereafter, 0.5 $\mathrm{ml}$ of sonic extract was injected subcutaneously until the precipitating activity of the serum with homologous sonic extract appeared maximal. Normal serum failed to produce precipitins.

Immunoprecipitation procedures. Double-diffusion-in-gel reactions were obtained by use of the method of Crowle (3) except that the agar contained a final concentration of $1 \%$ sodium azide instead of sodium chloride to prevent contamination.

Immunoelectrophoresis (IEP) was performed as previously described (2) on glass slides 1 by 3 inches [ca. 2.54 by $7.62 \mathrm{~cm}$ ], using Veronal buffer (pH 8.6 ; ionic strength, 0.075), $20 \mathrm{~V}$ per slide, and $46 \mathrm{~mA}$ for 80 min. Antiserum was added to cut out channels and was permitted to diffuse at room temperature towards separated antigen for $48 \mathrm{~h}$.

The FRIEP procedure used here is basically a modification of that described by Svendsen (9). The modifications included the use of elongated and overlapping antigen wells (Fig. 1), which facilitated the formation of lines of identity. Materials used included $30 \%$ antiserum in $1 \%$ agarose and Veronal buffer at $\mathrm{pH}$ 8.6 and $\theta .075$ ionic strength. Electrophoresis was permitted for $5 \mathrm{~h}$ at $2.7 \mathrm{~V} / \mathrm{cm}$ and $10 \mathrm{~mA}$. Results were photographed at $48 \mathrm{~h}$.

The acronym PQS (percent qualitative sharing) was developed as a means of relating organisms on the basis of antigen content. The PQS value is based on the total number of bands produced with a preparation homologous to the antiserum used in the study: PQS 
TABLE 1. Mycobacteria used in this study

\begin{tabular}{|c|c|c|c|}
\hline Species & Strain (serotype) & $\mathrm{TMC}^{a}$ no. & Special use \\
\hline M. tuberculosis & $\mathrm{H}_{37} \mathbf{R v}$ & 102 & Neotype \\
\hline M. kansasii ... & Bostrom & 1204 & Source of PPD-Y type strain \\
\hline M. intracellulare & Yandle (16) & 1406 & Type strain \\
\hline M. intracellulare . ..... & Boone (14) & 1403 & Source of PPD-B \\
\hline M. scrofulaceum . . . . . & Gause (43) & 1316 & Source of PPD-G \\
\hline M. scrofulaceum . ..... & La 2238 & 1323 & Type strain \\
\hline M. fortuitum . . . . . . & Cruz no. 1 & 1529 & Type strain \\
\hline$M$. fortuitum & Martin & 1530 & Source of PPD-F \\
\hline M. avium . . & $3459-1-\mathrm{T}(2)$ & 724 & Proposed neotype \\
\hline M. marinum & Aronson & 1218 & Type strain \\
\hline$M$. gordonae & P-15 & 1324 & Type strain \\
\hline$M . x e n o p i$ & Toad & 1470 & \\
\hline M. smegmatis . . . . . . & SW113 & 1546 & Type strain \\
\hline M. chelonei . . . . . . & Turtle & 1544 & Type strain \\
\hline M. phlei . . & Penso & 1523 & Proposed neotype \\
\hline
\end{tabular}

${ }^{a}$ TMC, Trudeau Mycobacterial Collection, Saranac Lake, N.Y.

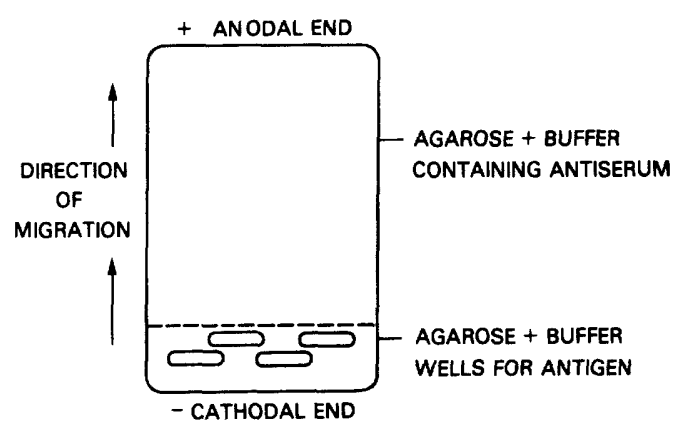

FIG. 1. Diagram of FRIEP procedure indicating antibody-containing layer, direction of antigen $\mathrm{mi}$ gration, and placement of antigen containing wells.

= (number of bands with heterologous preparation) number of bands with homologous preparation) $\times 100$. Currently, the method is qualitative and the detection of even a weak band is weighed as much as an intense band.

The crossed immunoelectrophoretic (CRIEP) procedure is a modification of that described by Weeke (10). Electrophoresis in the first dimension was accomplished as with IEP described above. For the second dimension the procedure was as with the FRIEP.

All photographs were taken the second day.

\section{RESULTS}

Figure 2 is a composite which illustrates advantages and disadvantages of various immunoprecipitation methodologies with antigen and antibody of the Gause strain of $M$. scrofulaceum. The simplest procedure, double diffusion in gel (Fig. 2A), permits the resolution of only about
10 lines of identity. The space between the wells of the reactants is usually small, and resolution of individual precipitate bands is difficult to visualize. Furthermore, this procedure may result in a balanced system for some antigens but not others, and some precipitates may form and be lost in the wells.

Conventional IEP procedure (Fig. 2B) separates the antigens on the basis of their electrophoretic mobilities. Antibody added after electrophoresis effected in essence double diffusion in gel with the separated antigens, and 20 bands were resolved. Although more antigens can be discerned with IEP, it is difficult to compare sharing of different antigens between organisms, and additional manipulations are required, such as the use of the Osserman technique (7).

CRIEP permits the greatest resolution with visualization of about 50 bands (Fig. 2C). Since this is a two-dimensional procedure, the formation of lines of identity with common antigens is also difficult (1).

Rocket IEP (RIEP) is also a very sensitive procedure although it is less sensitive than CRIEP in the development of discernible precipitin bands (Fig. 2D). However, a distinct advantage is gained over CRIEP in that when several sonic extracts are-set up in parallel with overlapping wells (Fig. 1), fusion of precipitin bands occurs between adjacent samples. This permitted a direct analysis of antigen sharing. This FRIEP procedure was, therefore, explored in preliminary serotaxonomic studies of mycobacteria.

Fig. 2. Comparison of four types of immunoprecipitation reactions in gels. (A) Double diffusion in gel, antigen on top, antiserum on bottom; (B) coventional IEP anode toward top of illustration; (C) RIEP; phoresis; (D) CRIEP. Antigen and antibody used were homologous for Mycobacterium scrofulaceum. 


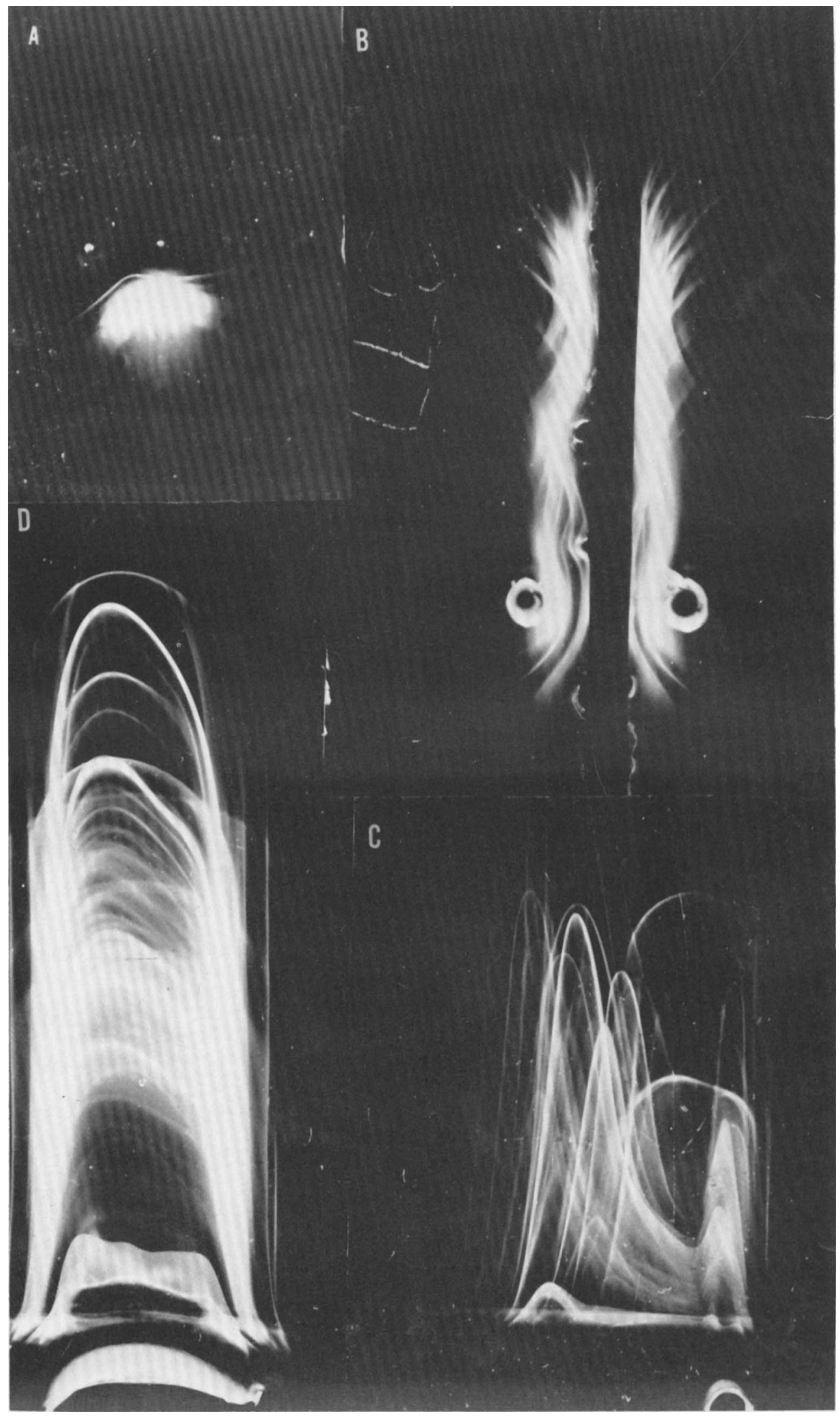


Figure 3 and Table 2 illustrate the usefulness of, and some of the problems associated with, FRIEP in the serotaxonomic analysis of strains of the same species. Sonic extracts of the type strain of M. fortuitum (Cruz No. 1) and of the strain which is the source of the widely used skin test antigen PPD-F (Martin) were compared. The inequity of the reagents became immediately apparent in that the antiserum produced toward the Cruz No. 1 strain had more precipitins than that of the Martin strain, whereas the sonic extract to the Martin strain had more demonstrable antigens. Nevertheless, a remarkable relationship could be established between the two strains. All the precipitable antigens discerned with Cruz No. 1 sonic extract could be related to corresponding antigens of the Martin strain. The Martin strain sonic extract had additional antigens demonstrable with both homologous and heterologous antisera. In fact,
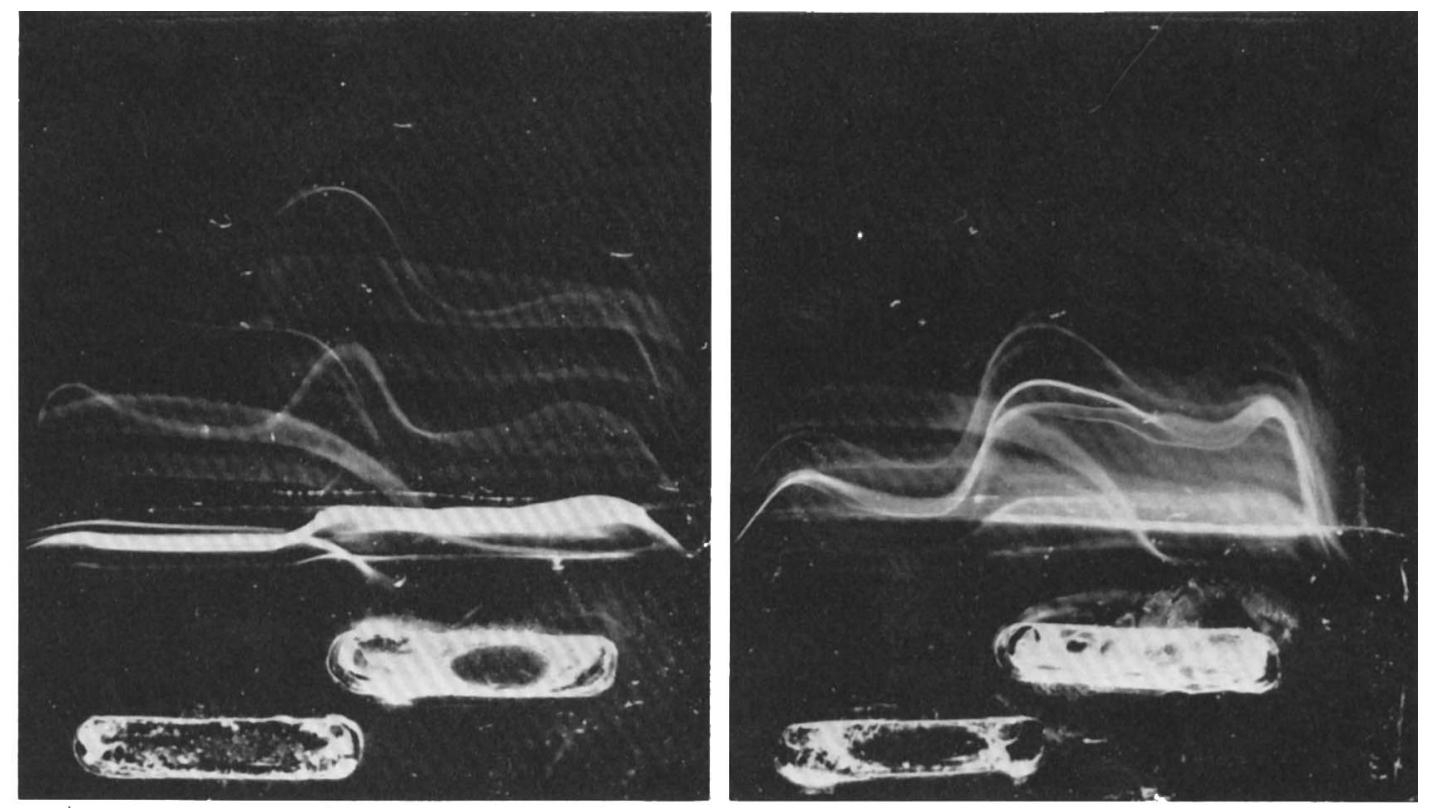

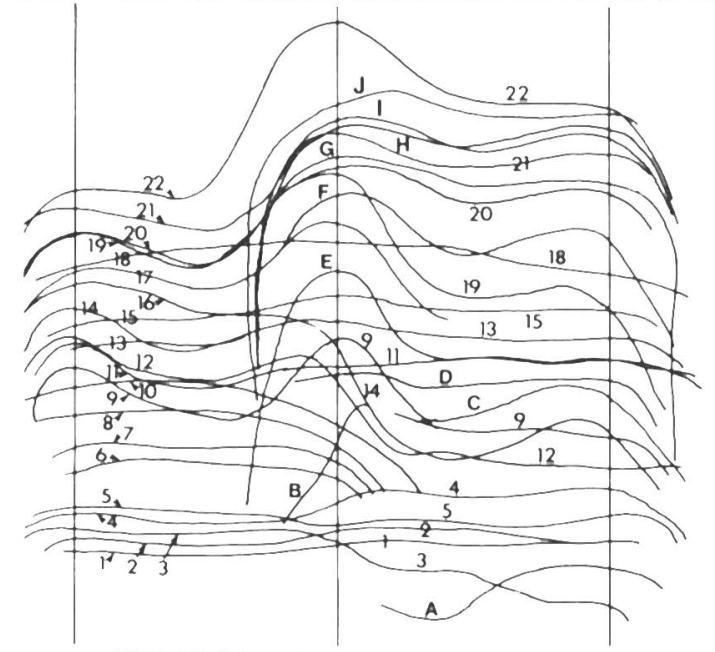

CRUZ NO. 1 MARTIN

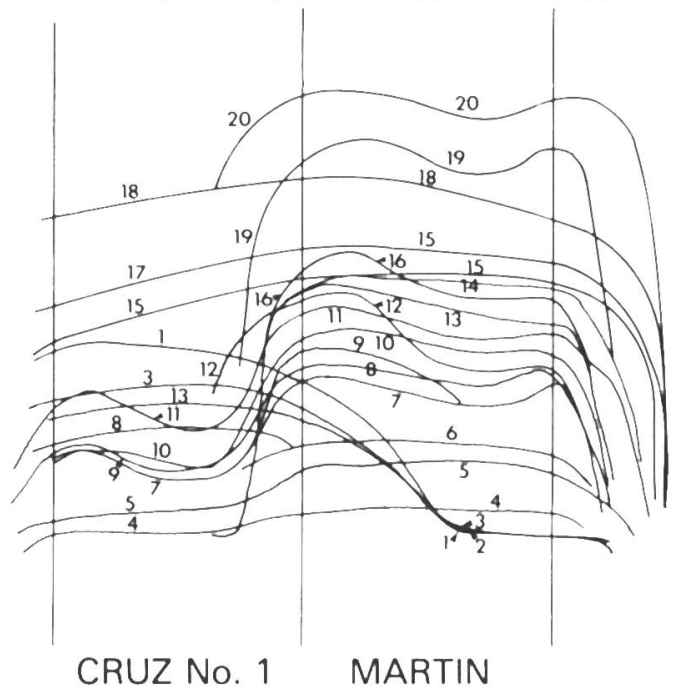

ANTI-MARTIN

ANTI-CRUZ No. 1

FIG. 3. Comparison of antigens in two strains of Mycobacterium fortuitum. 


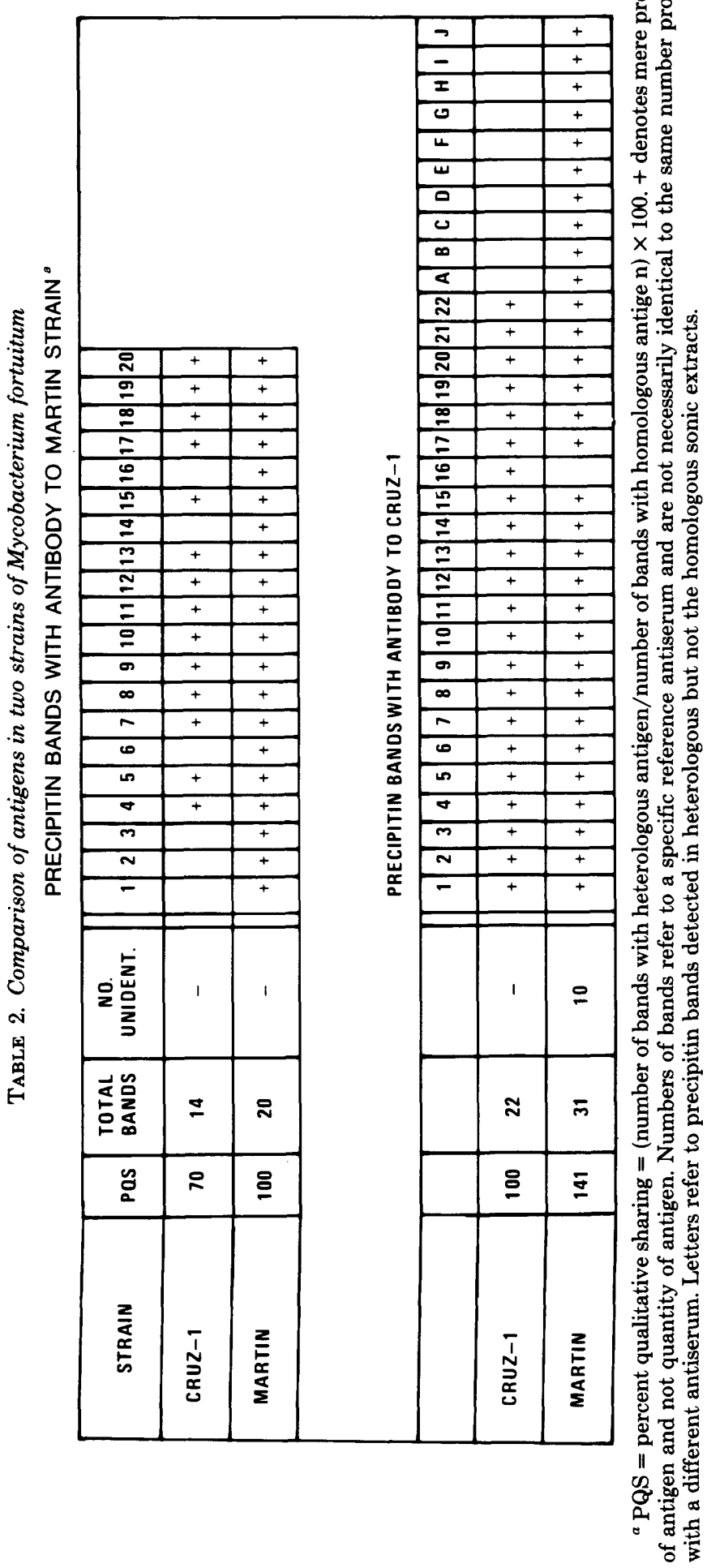


an additional 10 bands were noted with the heterologous antiserum for a total of 31 . Because of the large number of antigens associated with the Martin strain, a PQS of $141 \%$ was determined when the reference system was Cruz No. 1 sonic extract and Cruz No. 1 antiserum. Such a situation is encountered only occasionally and demonstrates the importance of the quality of the reagents. Nevertheless, the presence of a band implies that a specific determinant is produced by the organism and, if a high $P Q S$ value is obtained with a heterologous preparation, a high degree of relatedness is assumed.

Studies with two $M$. scrofulaceum strains showed a great deal of antigenic sharing (Fig. 4, Table 3). The antiserum and sonic extract for the La 2238 type strain were slightly more reactive than those for the Gause strain, the source strain for PPD-G skin test reagent. These organisms were highly related, with $P Q S$ values close to $100 \%$ in both systems.

Figure 5 and Table 4 indicate the close similarity between the type strain of $M$. intracellulare (Yandle), the proposed neotype strain of $M$. avium, and the Boone strain of $M$. intracellulare, which is used for the production of skin test antigen, PPD-battey. Antigenically, M. avium appears very similar to both strains of $M$. intracellulare, especially the Boone strain.

Figure 6 is illustrative of precipitin bands produced with seven species of mycobacteria developed with anti-M. fortuitum (Martin strain) serum. Antigen wells have been arranged so that each heterologous sonic extract is bordered by the homologous sonic extract. Frequently, additional slides or arrangements of reagents were made to confirm reported observations. A total of 22 bands were discernible with the homologous $M$. fortuitum sonic extract. Results are tabulated in Table 5. $M$. intracellulare crossreacted more $(\mathrm{PQS}=68 \%)$ than the other species. Band numbers $3,5,12$, and 20 were common to all sonic extracts tested. Bands 15 and 16 were common to almost all.

For the sake of brevity, illustrations of the following analyses have been omitted and only tables will be presented. Twenty-three bands were produced with $M$. scrofulaceum (La 2238) antiserum and the homologous sonic extract (Table 6). Bands 1 and 2 were present in all of the 7 sonic extracts except that of $M$. kansasii. Bands 3 and 6 were not detectable in $M$. intracellulare sonic extracts. $M$. gordonae displayed a high degree of relationship with $M$. scrofulaceum, with a PQS of $70 \%$. M. fortuitum, $M$. intracellulare, $M$. marinum, and $M$. tuberculosis all had PQS values of about 40\%. M. kansasii had a PQS of only $22 \%$.
In Table 7 are summarized the reactions elicited with anti- $M$. gordonae serum. Five of the seven heterologous organisms had PQS values around $50 \%$. The PQS in $M$. fortuitum was lower. Bands 1, 10, and 11 were found to be widely shared.

$M$. marinum antiserum produced 29 bands with its homologous sonic extract (Table 8). $M$. gordonae, M. tuberculosis, M. kansasii, M. xenopi, and $M$. smegmatis each produced from 11 to 13 bands with this antiserum.

The other organisms produced from seven to nine bands. Bands 1 and 8 were identifiable in all nine organisms tested. Antigens 2,3, and 6 were widely distributed in at least seven of the nine organisms.

\section{DISCUSSION}

FRIEP offered several advantages over the double-diffusion in-gel procedures of Ouchterlony and conventional IEP. FRIEP permitted the resolution of more bands than either of the other two procedures. Additionally, FRIEP allowed the separation of antigens on the basis of net charge on their molecules, and when several adjacent samples were subjected to electrophoresis simultaneously, lines of identity formed between antigens that had common determinants. These factors offered a great potential for serotaxonomic studies. As with all immunological procedures, several factors need to be considered. Because of the high sensitivity and resolving potential of the system, large numbers of antigens can be detected. However, it is often difficult to trace a precipitin band through a maze of other bands and relate it to a reference. Often it is necessary to alter the concentrations of reagents and the geometry of the wells. In the studies reported here, only uniformly produced sonic extracts were used in which denaturation was kept minimal. When culture filtrates or fractions were employed, the antigens could have been denatured to varying degrees and this may have affected the results.

Although FRIEP was a most useful adjunct in confirming or establishing relationships suggested by nonimmunological criteria, the comparison of more distantly related mycobacteria was more difficult. One reason for such difficulty is that often there are many and intense bands in the reference homologous system. Antigens present in heterologous preparations are often weak and may not peak at the same height as the corresponding homologous antigen. This sometimes results in failure to observe fusion of the lines and raises a question about their identities. Doubtlessly, some of these problems may be circumvented with the use of varying concen- 

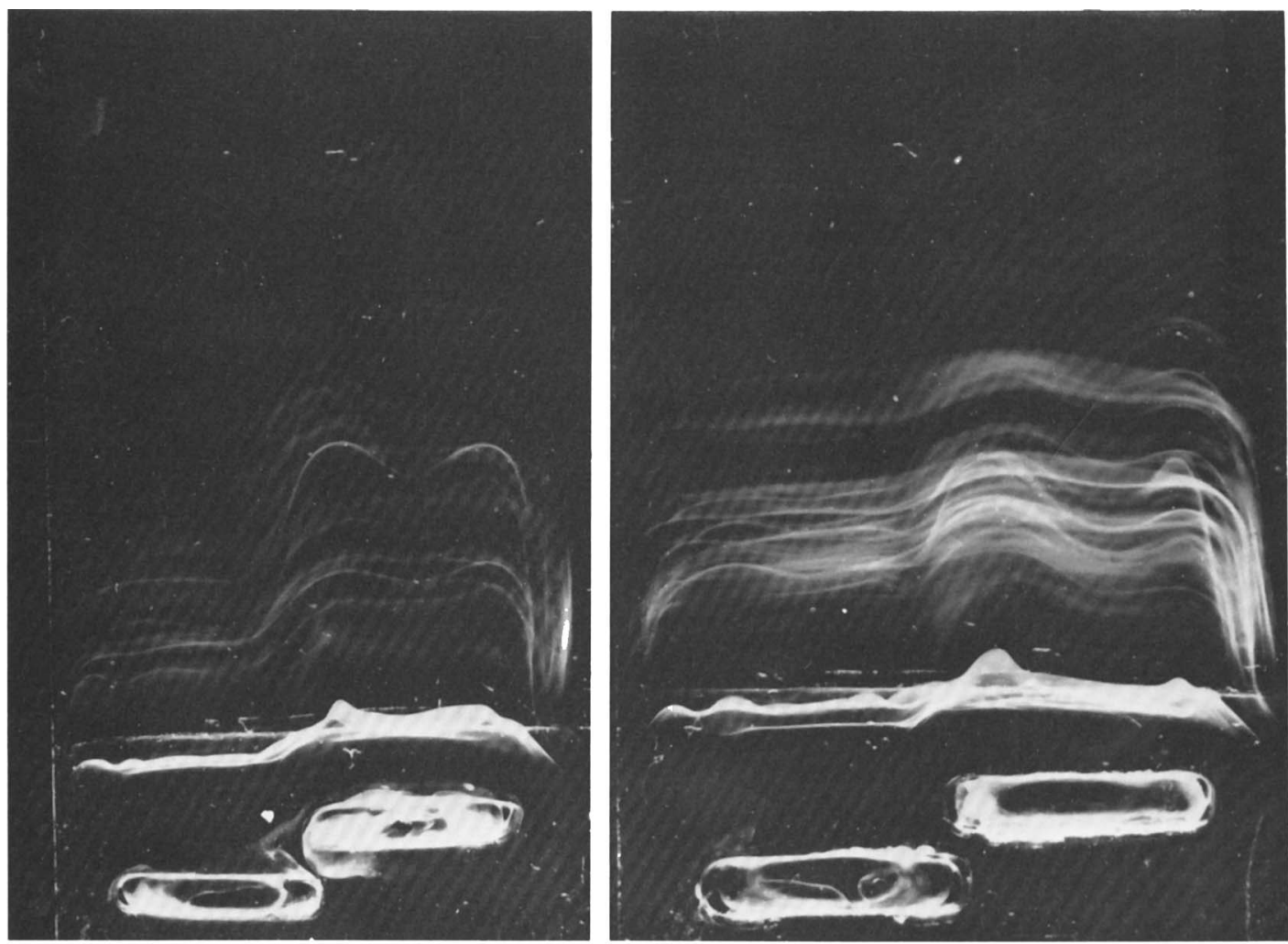

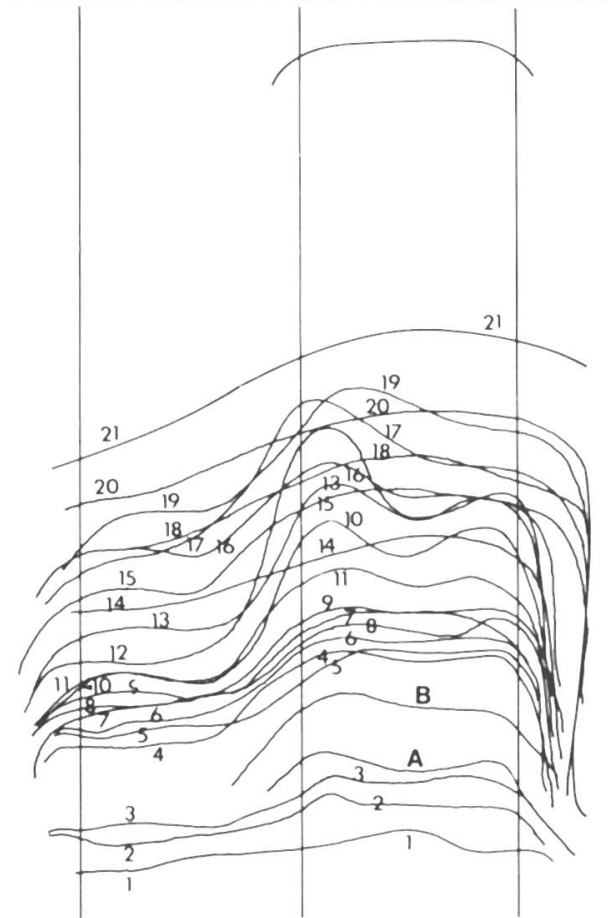

GAUSE LA-2238

ANTI-GAUSE

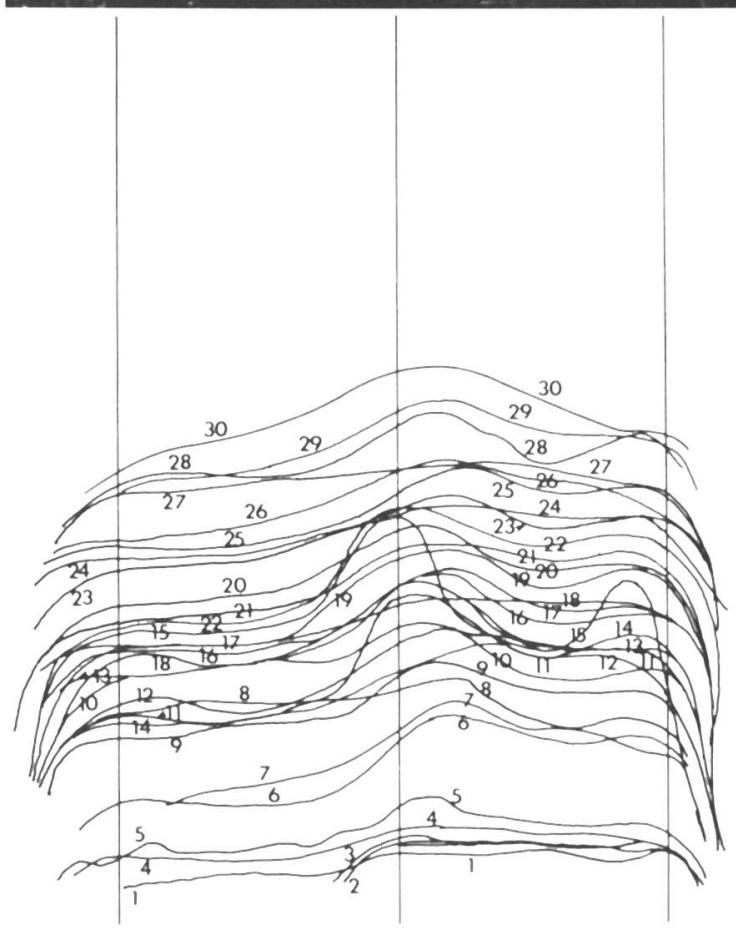

GAUSE
LA-2238

FIG. 4. Comparison of antigens in two strains of Mycobacterium scrofulaceum. 


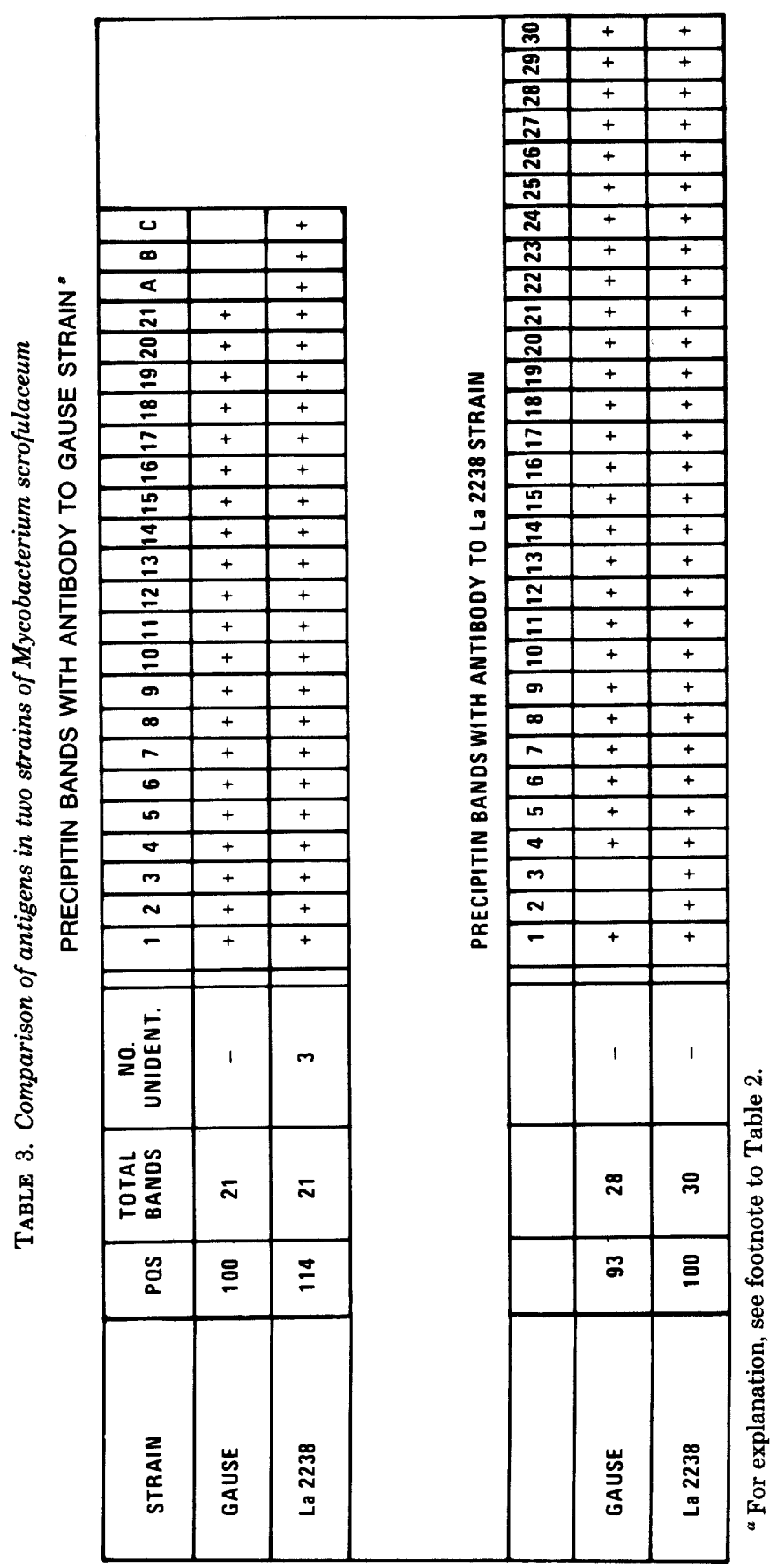



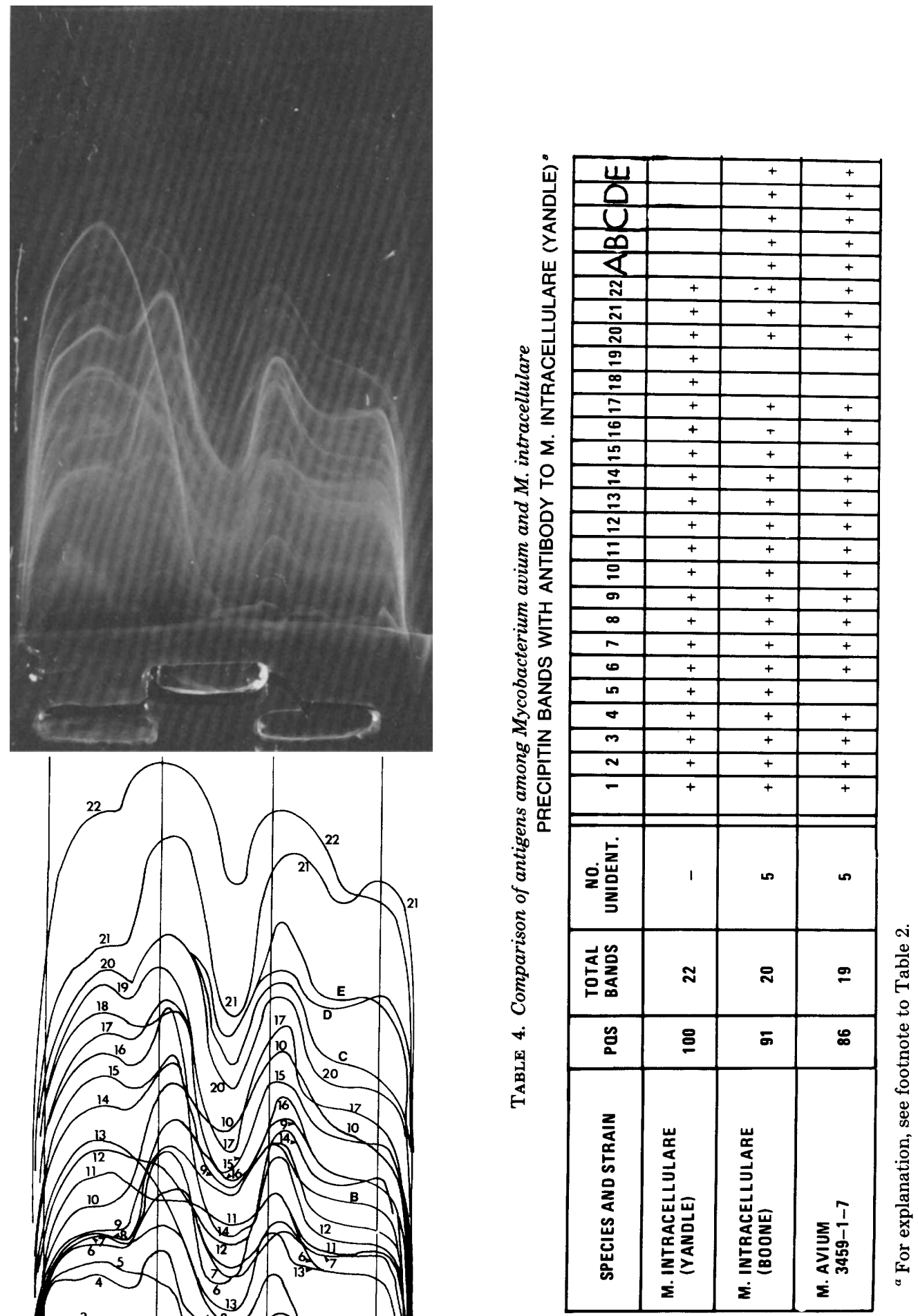

Fig. 5. Comparison of antigens among Mycobac-

INTR. BOONE

INTR. YANDLE AVIUM terium avium and $M$. intracellulare.

ANTI-INTRACELL. (YANDLE) 

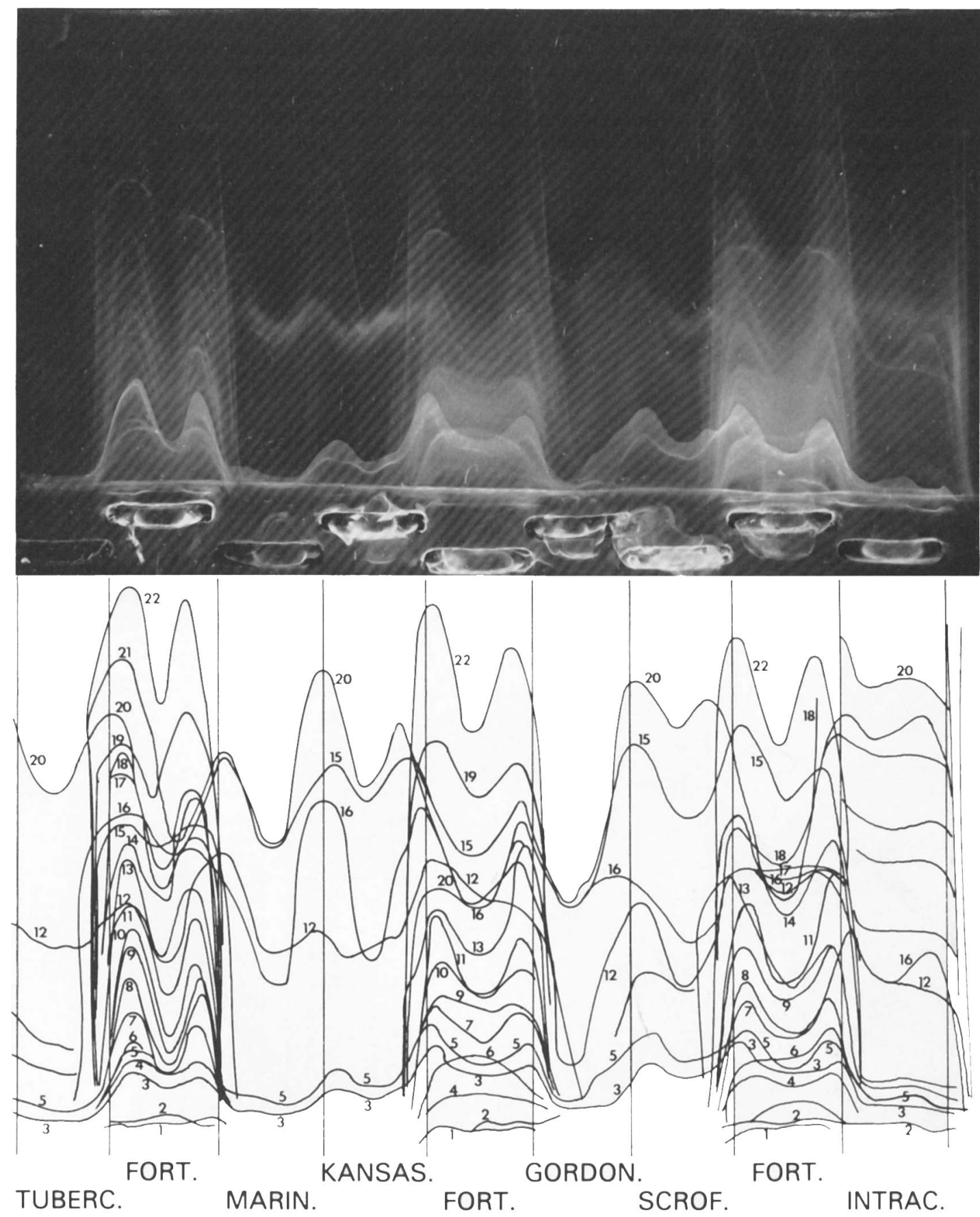

ANTI-FORTUITUM

FIG. 6. Precipitation reactions of various species of Mycobacterium with antibody to M. fortuitum. 
trations of reagents. One is, nevertheless, able to detect antigens which appear to be specific for each species. Isolation of such antigens may be followed to see whether such specificity is reflected in skin testing.

Band 8 developed with anti- $M$. marinum serum (Table 8 , figure not shown) was common to all nine mycobacterial species as judged from the test strains. The antigen responsible for this band was a minor component of the homologous $M$. marinum sonic extract but strikingly present in $M$. gordonae, $M$. xenopi, $M$. smegatis, and $M$. chelonii sonic extracts. Thus, minor but potent antigens in mycobacteria can stimulate good

TABLE 5. Comparison of antigens among various species of mycobacteria

PRECIPITIN BANDS WITH ANTIBODY TO $M$.

FORTUITUM (MARTIN) ${ }^{a}$

\begin{tabular}{|c|c|c|c|c|c|c|c|c|c|c|c|c|c|c|c|c|c|c|c|c|c|c|}
\hline $\begin{array}{l}\text { MYCOBACTERIAL } \\
\text { SPECIES }\end{array}$ & PQS & $\begin{array}{l}\text { TOTAL } \\
\text { BANDS }\end{array}$ & $\begin{array}{l}\text { NO. } \\
\text { UNIOENT. }\end{array}$ & 1 & 2 & 3 & 4 & 5 & 6 & 7 & 89 & & 11 & 12 & $13 \mid 14$ & 15 & 16 & $17 \mid$ & 1819 & 9 & & 22 \\
\hline $\begin{array}{l}\text { M. FORTUITUM } \\
\text { (MARTIN) }\end{array}$ & 100 & 22 & - & + & + & + & + & + & + & + & ++ & + & + & + & ++ & + & + & + & ++ & + & + & + \\
\hline M. INTRACELLULARE & 68 & 15 & 9 & & + & + & & + & & & & & & + & & & + & & & + & & \\
\hline M. SCROFULACEUM & 32 & 7 & 1 & & & + & & + & & & & & & + & & + & + & & & + & & \\
\hline M. GORDONAE & 27 & 6 & - & & & + & & + & & & & & & + & & + & + & & & + & & \\
\hline M.KANSASII & 27 & 6 & - & & & + & & + & & & & & & + & & $1+$ & + & & & + & & \\
\hline M. MARINUM & 27 & 6 & - & & & + & & + & & & & & & + & & $1+$ & + & & & + & & \\
\hline M. TUBERCULOSIS & 27 & 6 & 2 & & & + & & + & & & & & & + & & & & & & + & & \\
\hline
\end{tabular}

${ }^{a}$ For explanation, see footnote to Table 2.

TABLE 6. Comparison of antigens among various species of mycobacteria PRECIPITIN BANDS WITH ANTIBODY TO $M$. SCROFULACEUM (L22238) ${ }^{\circ}$

\begin{tabular}{|c|c|c|c|c|c|c|c|c|c|c|c|c|c|c|c|c|c|c|c|c|c|c|c|}
\hline $\begin{array}{l}\text { MYCOBACTERIAL } \\
\text { SPECIES }\end{array}$ & PAS & $\begin{array}{l}\text { TOTAL } \\
\text { BANDS }\end{array}$ & $\begin{array}{l}\text { NO. } \\
\text { UNIDENT. }\end{array}$ & 1 & 2 & 3 & 4 & 5 & 6 & 7 & 8 & $9 \mid 1$ & 1011 & 1112 & 13 & $14 \mid 1$ & $15 \mid 16$ & $17 \mid$ & $18 \mid 1$ & 1920 & 202 & 212 & 223 \\
\hline M. SCROFULACEUM & 100 & 23 & - & + & + & + & + & + & + & + & + & + & ++ & ++ & + & + & ++ & + & + & ++ & + & ++ & ++ \\
\hline M. GORDONAE & 70 & 16 & 3 & + & + & + & & & + & & & + & & & & & + & + & + & ++ & ++ & + & ++ \\
\hline M. FORTUITUM & 43 & 10 & 1 & + & + & + & & & + & & + & + & & + & & & & & & + & & & + \\
\hline M. INTRACELLULARE & 39 & 9 & 6 & + & + & & & & & & + & & & & & & & & & & & & \\
\hline M. MARINUM & 39 & $\mathbf{g}$ & - & + & + & + & & & + & + & & + & & & & + & & & & & & & ++ \\
\hline M. TUBERCULOSIS & 35 & 8 & 1 & + & + & + & & & + & & & & + & & + & & & & & & & + & \\
\hline M. KANSASII & 22 & 5 & - & & & + & & & + & + & & & & & & $1+$ & & & & & & & + \\
\hline
\end{tabular}

${ }^{a}$ For explanation, see footnote to Table 2 . 
TABLE 7. Comparison of antigens among various species of mycobacteria

PRECIPITIN BANDS WITH ANTIBODY TO $M$. GORDONAE *

\begin{tabular}{|c|c|c|c|c|c|c|c|c|c|c|c|c|c|c|c|c|c|c|}
\hline $\begin{array}{l}\text { MYCOBACTERIAL } \\
\text { SPECIES }\end{array}$ & PaS & $\begin{array}{l}\text { TOTAL } \\
\text { BANDS }\end{array}$ & $\begin{array}{l}\text { NO. } \\
\text { UNIDENT. }\end{array}$ & 1 & 2 & 3 & 4 & 5 & 6 & 7 & 8 & 9 & 10 & 11 & $12 \mid 1$ & $13 \mid 1$ & $14 \mid 1$ & 51 \\
\hline M. GORDONAE & 100 & 16 & - & + & + & + & + & + & + & + & + & + & + & + & + & + & ++ & ++ \\
\hline M. INTRACELLULARE & 56 & 9 & 4 & + & & & & & & & & & + & + & & & & ++ \\
\hline M. SCROFULACEUM & 56 & 9 & 2 & + & & & & & & + & & & + & + & & & ++ & ++ \\
\hline M. KANSASII & 50 & 8 & 2 & + & & & & + & + & + & & & + & + & & & & \\
\hline M. TUBERCULOSIS & 44 & 7 & 4 & & & & & & + & & & + & & + & & & & \\
\hline M. MARINUM & 44 & 7 & 1. & + & & & & + & + & + & & & + & + & & & & \\
\hline M. FORTUITUM & 6 & 2 & - & & & & & & & & & & + & + & & & & \\
\hline
\end{tabular}

${ }^{a}$ For explanation, see footnote to Table 2 .

immunological responses toward major heterologous antigens. This fact may also impede investigations concerned with specificity of mycobacterial antigens.

The PQS was used to express relatedness to the homologous system. Even though lines of identity were not established for all antigens, the formation of a precipitin band to a heterologous preparation with a reference antiserum most likely means that the heterologous antigen shares determinants with the homologous antigen to which the antibody was raised. It should be stressed that these are minimal numbers of shared antigens and, with more concentrated preparations or different arrangements of antigenic preparations, more bands might be detectable. The extensive sharing of antigens prompted Stanford (8) to suggest that the various species of Mycobacterium may have developed from a common progenitor through a series of mutations. It might be expected then that certain stable structures may have remained common to the group. It is also possible that mutation may affect only a portion of a molecule and that, although certain determinants may be unique, common core structures may be shared. Such a situation may have confounded the many efforts to isolate monospecific antigens from $M$. tuberculosis.
The preliminary serotaxonomic studies presented here underscore the extent of antigen sharing among the mycobacteria. In many instances, certain bands could be referred to by a numbering scheme. It would be of great value to produce and select large batches of antisera and antigenic preparations for type strains to be used as reference systems by investigators actively exploring antigenic relatedness of mycobacteria.

It is comforting to establish a close relationship between the source strains for production of widely used skin test antigens, $M$. fortuitum and $M$. scrofulaceum and for their corresponding type strains. Even though the reagents used were not balanced, the PQS values indicate very strong antigenic sharing.

The taxonomic status of $M$. avium and $M$. intracellulare is still under dispute (6). When a sonic extract of the proposed neotype $M$. avium was compared to the type strain of $M$. intracellulare, and to the strain used to prepare the Battey skin test antigen PPD-B, the patterns observed suggested virtual antigenic identity of the two species.

The potential utility of FRIEP is thus apparent. Further work needs to be done to increase its utility, such as the standardization of reagents and the improvement of procedures based on quantitative assessment of antigenic sharing. 


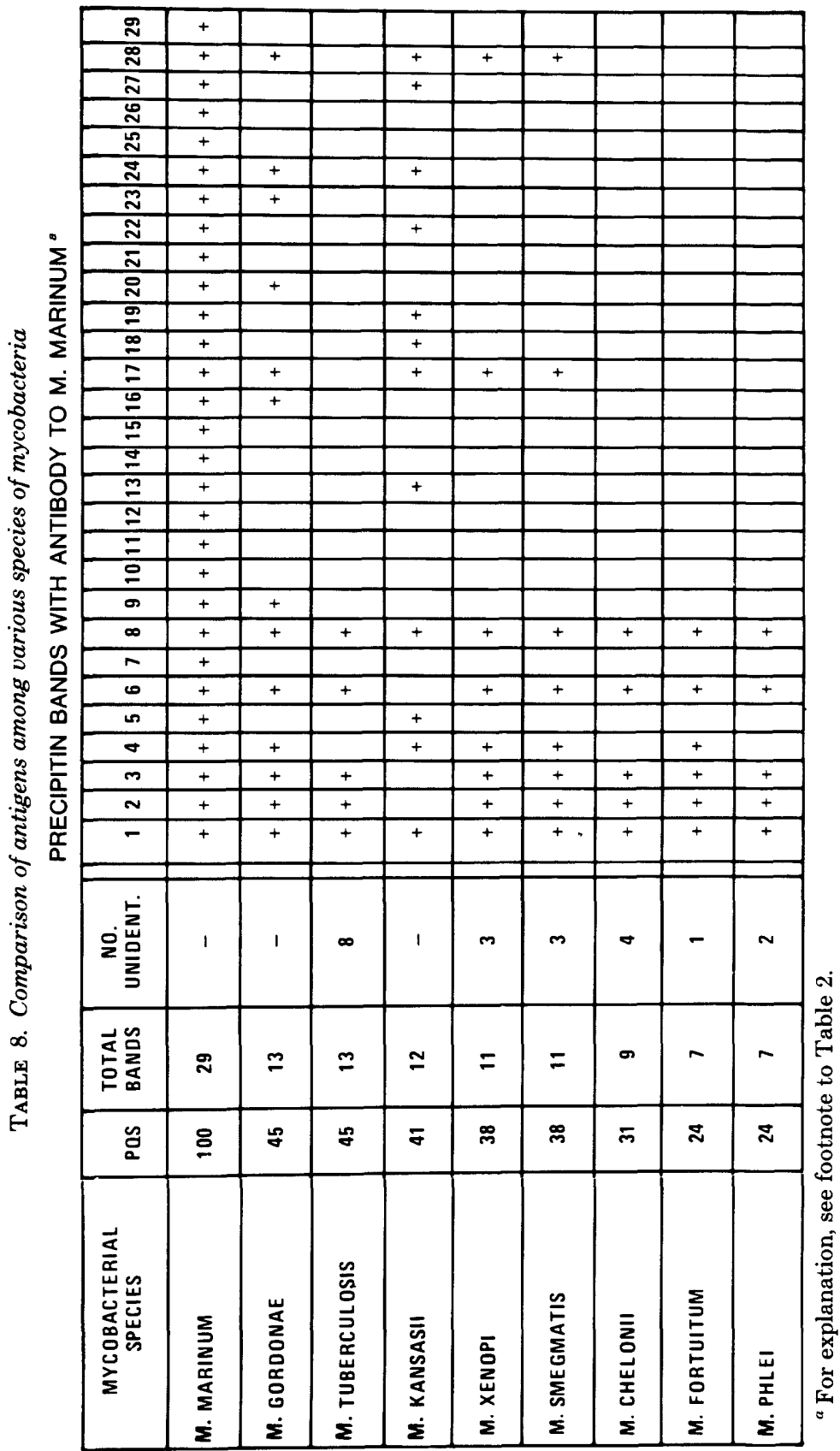




\section{REPRINT REQUESTS}

Address reprint requests to: Dr. Sotiros D. Chaparas, Bureau of Biologics, Food and Drug Administration, Bethesda, MD 20014.

\section{LITERATURE CITED}

1. Axelsen, N., H. E. Bock, and S. Kroll. 1973. Comparison of antigens: the reaction of identity. In A manual of quantitative immunoelectrophoresis. Scand. J. Immunol. 2(Suppl. 1):91-94.

2. Chaparas, S. D., and S. R. Hedrick. 1973. Comparison of strains of BCG. I. Antigenic analysis and tuberculin reactivity. Infect. Immun. 1:777-780.

3. Crowle, A. J. 1958. A simplified micro double-diffusion agar precipitin technique. J. Lab. Clin. Med. 52:784-787.

4. Janicki, B. W., S. D. Chaparas, T. M. Daniel, G. P. Kubicka, G. L. Wright, and G. S. Yee. 1971. A reference system for antigens of Mycobacterium tuberculosis. Am. Rev. Resp. Dis. 104:602-604.

5. Long, E. R. 1958. The chemistry and chemotherapy of tuberculosis, chapter 2, p. 24. The William and Wilkins Co., Baltimore.

6. Meissner, G., et al. 1974. A co-operative numerical analysis on nonscoto- and nonphoto-chromogenic slowly growing mycobacteria. J. Gen. Microbiol. 83:207-235.

7. Osserman, E. F. 1960 . A modified technique of immunoelectrophoresis facilitating the identification of specific precipitin arcs. J. Immunol. 84:93-97.

8. Stanford, J. L. 1973. Immunodiffusion analysis-a rational basis for the taxonomy of mycobacteria. Ann. Soc. Belge. Med. Trop. 53:321-330.

9. Svendsen, P. J. 1973. Fused rocket immunoelectrophoresis. In A manual of quantitative immunoelectrophoresis. Scand. J. Immunol. 2(Suppl. 1):69-70.

10. Weeke, B. 1973. Crossed immunoelectrophoresis. In A manual of quantitative immunoelectrophoresis. Scand. J. Immunol. 2 (Suppl. 1):47-56. 